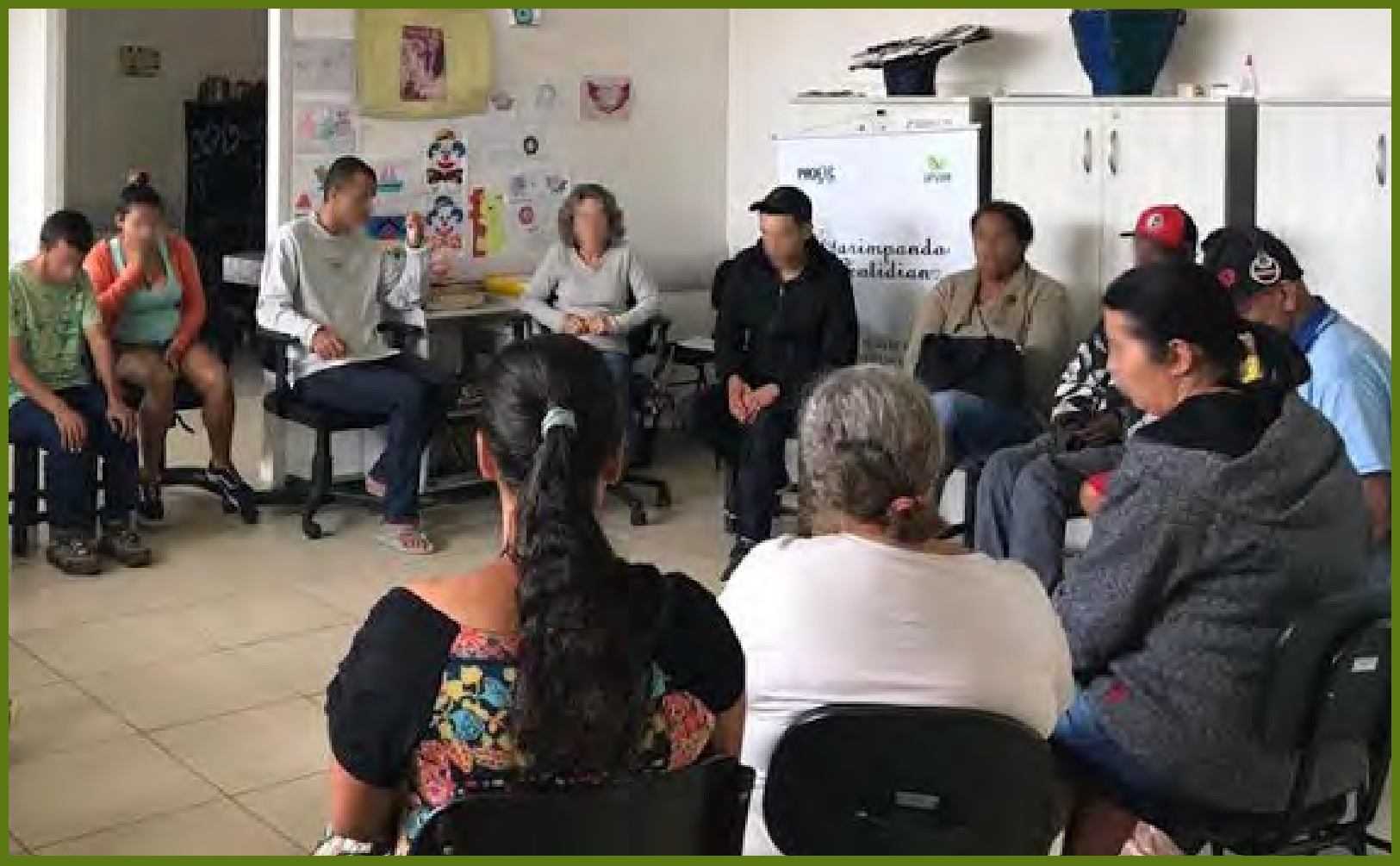

\title{
Cuidar da saúde mental "Garimpando o Cotidiano": grupos terapêuticos em um Centro de Atenção Psicossocial
}

Letícia Perígolo Jorge - leticiaperigolo@hotmail.com¹

Roberta Vasconcelos Leite - roberta.leite@ufvjm.edu.br²

\section{RESUMO}

O projeto de extensão "Garimpando o Cotidiano" realiza grupos de elaboração da experiência para promover saúde mental em Diamantina - MG, embasado na Fenomenologia. Em 15 meses, foram realizados 19 encontros no Centro de Atenção Psicossocial - CAPS Renascer, com 323 participantes. Identificam-se duas fases dos grupos neste período, que demonstram amadurecimento. Conclui-se que a proposta de atenção a acontecimentos cotidianos como estratégia de cuidado tem mobilizado transformações nos participantes.

PALAVRAS-CHAVE: saúde mental; grupos terapêuticos; fenomenologia.

1 Graduanda em Medicina pela Universidade Federal dos Vales do Jequitinhonha e Mucuri.

2 Psicóloga e doutora em Psicologia pela Universidade Federal de Minas Gerais. Professora adjunta da Faculdade de Medicina da Universidade Federal dos Vales do Jequitinhonha e Mucuri. 


\title{
Taking care of mental health "Panning the Daily Life": therapeutic groups in a Psychosocial Care Center
}

\begin{abstract}
The extension project "Garimpando o Cotidiano" [Panning the Daily Life] holds groups to elaborate the daily life experience to further mental health in Diamantina - MG, based on Phenomenology. In 15 months, 19 meetings were held at the Psychosocial Care Center - CAPS Renascer, with 323 participants. Two phases of the groups are identified in this period, which demonstrate growth maturation. The conclusion holds that proposing attention to everyday events as a care strategy has mobilized a transformation in participants' lives.
\end{abstract}

KEYWORDS: mental health; therapeutic groups; phenomenology.

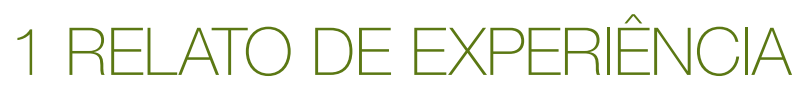

Embora fortemente negligenciada, a saúde mental é fundamental no conceito de saúde da Organização Mundial de Saúde (OMS, 2002). Nos tempos pós-modernos, marcados por fluidez, multiplicam-se quadros de depressão e ansiedade e crescem taxas de suicídio, desafios que podem ser associados ao distanciamento da própria subjetividade e da relação com o outro (MENDES LEMOS; CELIO FREIRE, 2011).

Nesse cenário, o modelo biomédico em saúde mental tem na medicalização seu principal recurso terapêutico, o que se mostra insuficiente dada a complexidade dos processos de adoecimento e os efeitos iatrogênicos do uso exacerbado de psicotrópicos (BRASIL, 2015). Por outro lado, iniciativas alinhadas à Reforma Psiquiátrica buscam alternativas que reconheçam a singularidade dos indivíduos em sofrimento mental (ISHARA; CARDOSO; LOUREIRO, 2013). Valorizando trabalhos grupais, buscam criar espaços comunitários que favoreçam trocas de saberes e que propiciem empatia e resiliência (ROCHA, 2015).

Consoanteaesta segundavertente, o projeto de extensão "Garimpando o cotidiano: cultura, experiência e promoção da saúde mental", vinculado à Faculdade de Medicina de Diamantina da Universidade Federal dos Vales do Jequitinhonha e Mucuri ${ }^{3}$, agrega docentes e discentes que buscam construir uma perspectiva interdisciplinar que contemple conhecimentos da psicologia, psiquiatria, educação popular e filosofia. Seu objetivo é realizar grupos de elaboração coletiva das experiências cotidianas, tendo como fundamentação teórica a Fenomenologia Clássica (STEIN, 2005), corrente que preconiza a atenção à experiência e reconhece a centralidade da dimensão relacional e dos vínculos comunitários na constituição do ser humano.

Inspirados na metodologia dos Grupos Comunitários de Saúde Mental de Ribeirão Preto (ISHARA; CARDOSO; LOUREIRO, 2013) e da Terapia Comunitária Integrativa (BARRETO, 2008), todos os encontros são abertos, sem compromisso de continuidade e integram momentos culturais (apresentação de músicas, textos, filmes, ou imagens considerados belos) e trocas de vivências (acontecimentos cotidianos e reverberações pessoais).

Os grupos têm sido realizados tanto na universidade quanto em instituições de saúde da cidade, como os Centros de Atenção Psicossocial (CAPS), hospitais e centro de reabilitação. A integração com o ensino se dá por meio da realização de grupos com discentes de diferentes cursos e pela proposição da participação em pelo menos um grupo no CAPS como atividade do quinto período do curso de Medicina. A integração com a pesquisa tem sido construída em parcerias com grupos da UFVJM que se dedicam a questões relativas à saúde mental, como o "Grupo Vida-Suicidologia" e "Geografia Humanista, Arte e Psicologia Fenomenológica" (Ghuapo).

Neste artigo, são apresentados resultados referentes aos encontros ocorridos de outubro de 2018 a dezembro de 2019 no CAPS Renascer de Diamantina (Figura 1). Participaram dessas reuniões usuários do serviço, seus familiares, funcionários da instituição, discentes e docentes do curso de Medicina. A condução foi realizada pela professora coordenadora do projeto e pela discente bolsista.

30 projeto foi contemplado com bolsas de extensão da Pró-reitoria de Extensão da UFVJM para o período de fevereiro/2019 a dezembro/2021 (Editais Pibex 01/2019, 01/2020 e 01/2021). 
Figura 1 - Entrada do CAPS Renascer

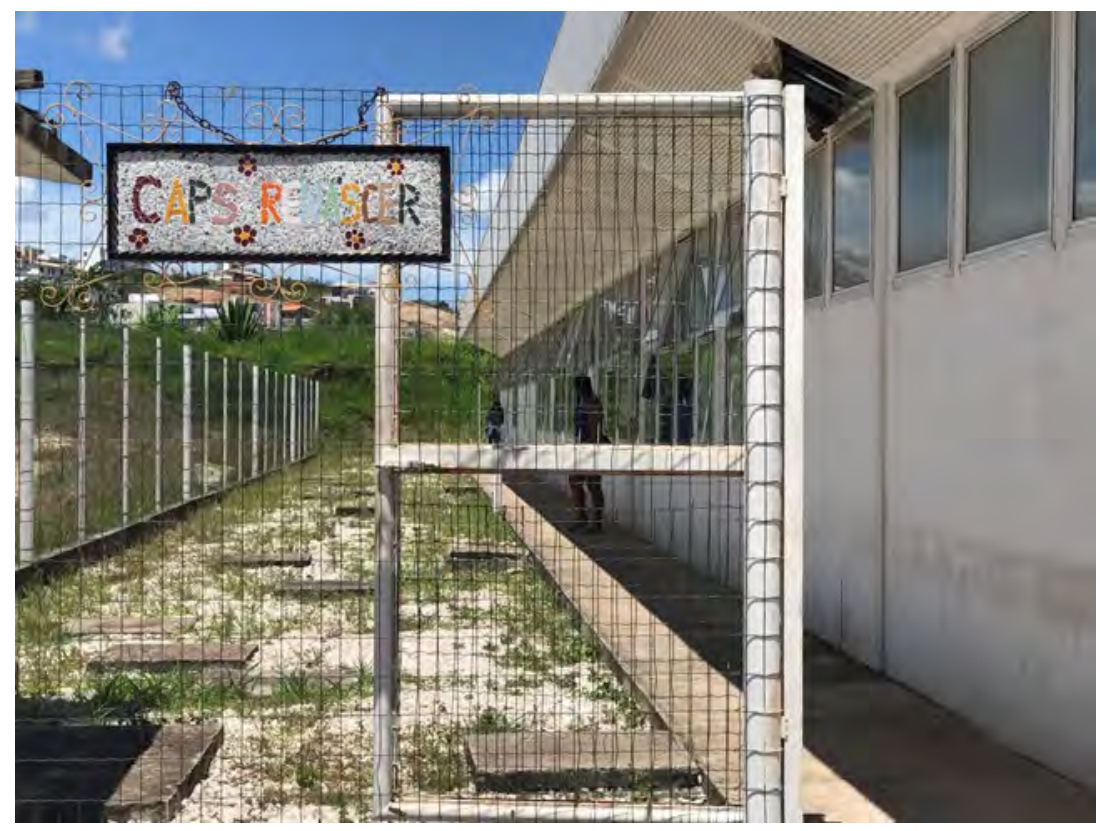

Fonte: Arquivos do projeto.

A dinâmica dos encontros revela duas fases do projeto, sendo a primeira de outubro/2018 a agosto/2019, e a segunda de setembro a dezembro/2019. Na primeira fase (Figura 2), os encontros aconteciam no mesmo dia das consultas com a psiquiatra, havendo sempre grande fluxo de pessoas, o que se mostrou desfavorável à participação regular. Foi possível observar efeitos positivos, como o sentimento de empatia entre os participantes que buscavam se ajudar mutuamente diante dos problemas expostos.

\section{Figura 2 - Grupo "Garimpando o Cotidiano" no CAPS Renascer em 13/05/2019}

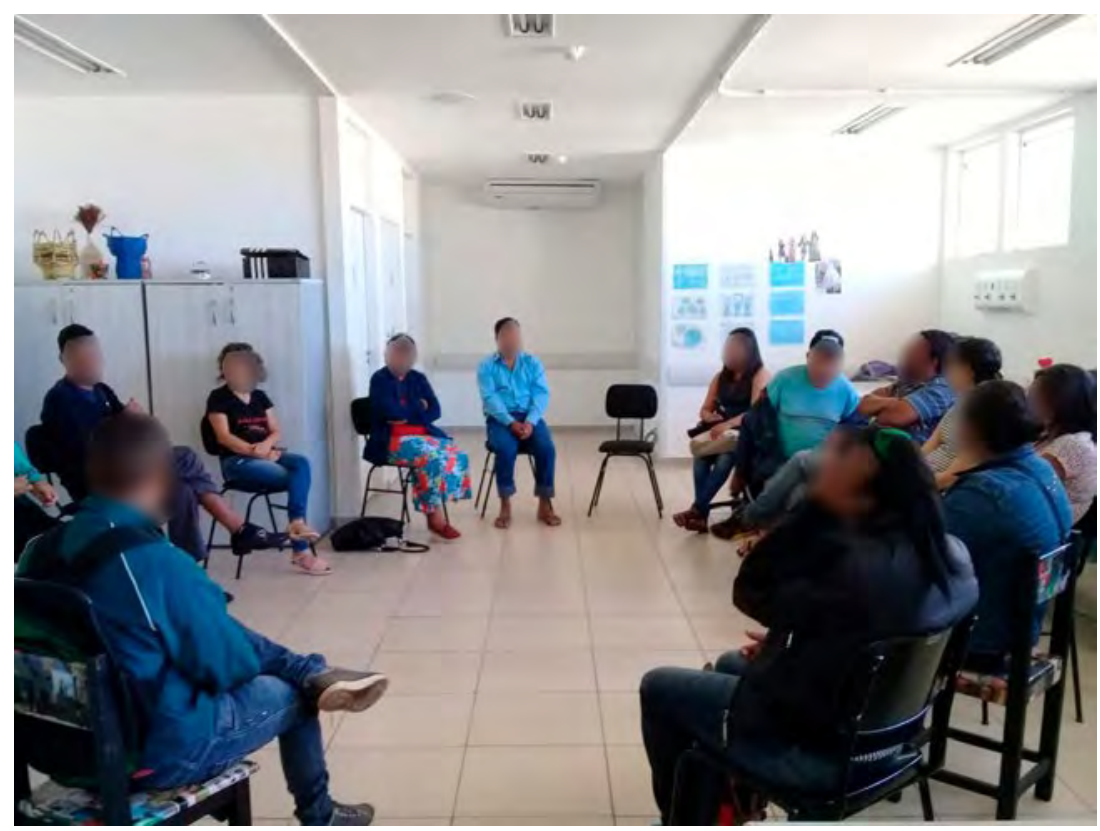

Fonte: Arquivos do projeto.

Na segunda fase (Figura 3), os grupos já apresentavam contornos mais bem estabelecidos, e o dia dos encontros foi oportuno para favorecer a regularidade de participação dos usuários, o que maximizou seu potencial terapêutico. 
Figura 3 - Grupo "Garimpando o Cotidiano" no CAPS Renascer em 10/12/2019.

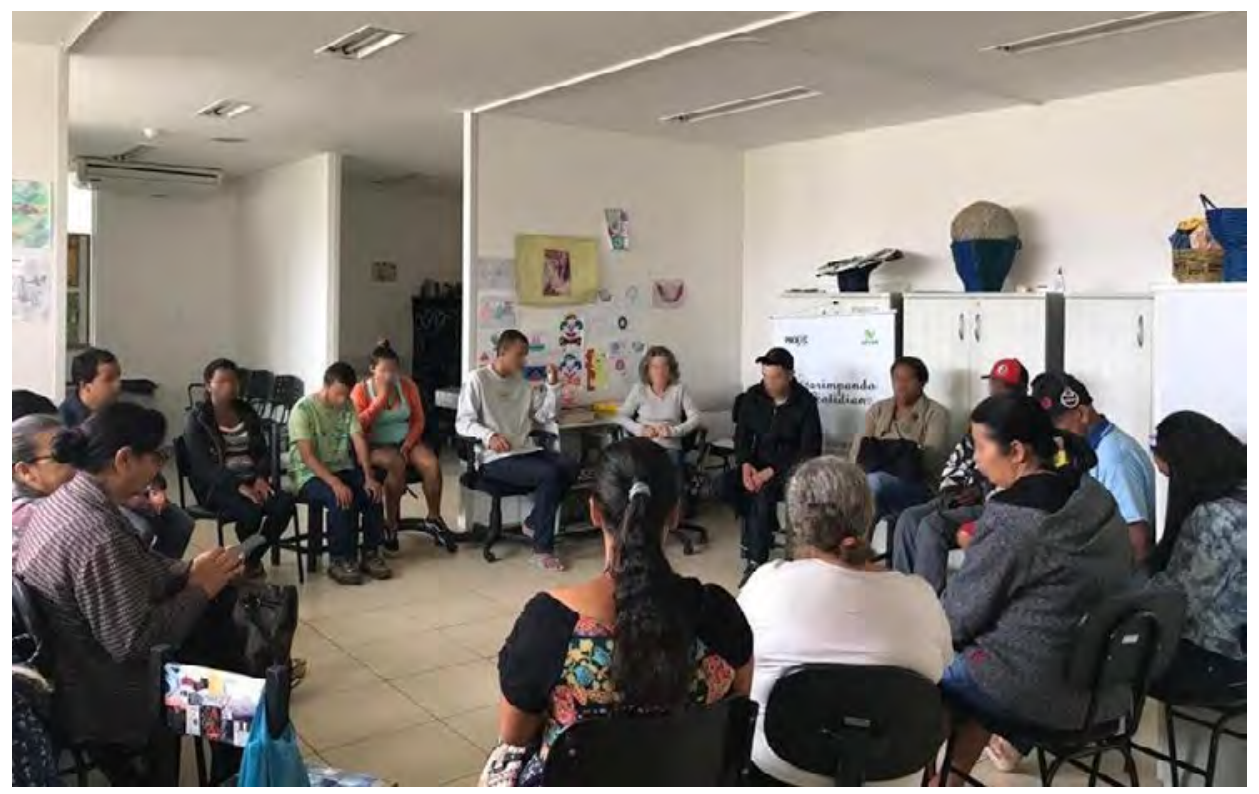

Fonte: Arquivos do projeto.

Foram percebidos sinais de amadurecimento na dinâmica grupal durante esse período: preparo antecipado dos participantes para os encontros e comprometimento voluntário de presença; liberdade para expressar vivências difíceis; consolidação dos grupos como proposta do CAPS; teor resiliente das falas; corresponsabilidade pelo bom funcionamento dos grupos assumida por participantes regulares. Esses fatores revelam-se consoantes com resultados encontrados por Pinheiro (2017) ao investigar os Grupos Comunitários de Saúde Mental de Ribeirão Preto.

Pôde-se observar ainda que indivíduos mais tímidos encontraram espaço ao longo do tempo para partilhar suas vivências, à medida em que se sentiram pertencentes ao grupo. Nessa decisão de fala, ocorreu uma legitimação da própria experiência pela pessoa, que outrora poderia não considerá-la relevante o suficiente para ser compartilhada, assim como descrito por Rocha (2015).

0 impacto do projeto em termos quantitativos é expressivo: em 15 meses foram organizados 19 grupos, com 323 participantes. Para além desses dados mensuráveis, os depoimentos ao fim dos encontros apontam os grupos como propulsores de novas formas de lidar com dificuldades e vivências no dia a dia, mobilizando transformações na própria vida dos participantes e na vida daqueles com que convivem. Alguns exemplos: "Enquanto existir CAPS, tem que existir esse grupo", "V. melhorou e diminuiu suas crises depois do grupo"; "Ajuda quem é tímido, estimula a falar, a ouvir"; "Cheguei vazio, mas estou saindo daqui cheio".

Destaca-se que o mesmo processo pode ser percebido nos estudantes de Medicina, como sintetizado por uma aluna:

É perceptível como os participantes reconhecem no grupo um espaço confiável, expressando aspectos que habitualmente não são compartilhados tão facilmente com pessoas transvestidas de médicos através dos seus jalecos brancos. $\dot{E}$ também admirável se certificar, de corpo presente, que todas as pessoas, mesmo as mais oprimidas e marginalizadas, acumulam significativos e surpreendentes saberes para a solução dos seus problemas e melhorias das suas vidas.

Este depoimento sinaliza como os grupos "Garimpando o Cotidiano" estimulam vivências de elaboração da experiência numa perspectiva horizontal, ao abrir espaço para que pessoas provenientes de vários setores da sociedade sejam cuidados e cuidadores uns dos outros. Reconhecendo o caráter inicial e limitado das análises apresentadas, pode-se concluir que a proposta de atenção a acontecimentos cotidianos como estratégia de cuidado tem mobilizado transformações nos participantes, principalmente daqueles que frequentam as reuniões regularmente, sendo provavelmente esses os maiores beneficiados. Dessa forma, foi possível verificar como essa metodologia traz efeitos significativos para promoção da saúde mental dos participantes. Dado seu potencial para promover saúde mental da população de Diamantina, espera-se que mais pesquisas possam documentar seus desdobramentos. 


\section{REFERÊNCIAS}

BARRETO, Alberto. Terapia comunitária: passo a passo. 3.ed. Fortaleza: LCR, 2008.

BRASIL. Ministério da Saúde. Caderno HumanizaSUS: saúde mental. Brasília: Secretaria de Atenção à Saúde, 2015.

ISHARA, Sergio; CARDOSO, Carmen Lúcia; LOUREIRO, Sonia Regina. Grupo comunitário de saúde mental: conceito, delineamento metodológico e estudos. Ribeirão Preto: Nova Enfim, 2013.

MENDES LEMOS, Patrícia; CELIO FREIRE, José. Os contornos tardo-modernos do sofrimento e do adoecimento psíquico: proposições éticas para o Centro de Atenção Psicossocial. Psicologia em revista, Belo Horizonte, v. 17, n. 2, 2011, p.303-321.

ORGANIZAÇÃO MUNDIAL DE SAÚDE. Relatório mundial da saúde: saúde mental: nova concepção, nova esperança. Lisboa: OMS, 2002.

PINHEIRO, Bruna Cardoso. Grupo Comunitário de Saúde Mental: formação de Recursos Humanos. 2017. 179 f. Dissertação (Mestrado) - Faculdade de Filosofia, Ciências e Letras de Ribeirão Preto, Universidade de São Paulo, Ribeirão Preto, 2017.

ROCHA, Rita Martins Godoy. Análise compreensiva de uma nova modalidade de trabalho em saúde: 0 Grupo Comunitário de Saúde Mental. 2015. 161 F. Tese (Doutorado) - Faculdade de Filosofia, Ciências e Letras de Ribeirão Preto, Universidade de São Paulo, Ribeirão Preto, 2015.

STEIN, Edith. Contribuiciones a la fundamentación filosófica de la psicología y de las ciencias del espíritu. In STEIN, Edith. Obras completas. v.ll: escritos filosóficos (etapa fenomenológica: 1915-1920). Trad. F. J. Sancho e col. Burgos: Monte Carmelo, 2005, p. 207-520. 